\title{
Pseudo almost periodic solutions for neutral type high-order Hopfield neural networks with mixed time-varying delays and leakage delays on time scales*
}

\author{
Yongkun $\mathrm{Li}^{a \dagger}$, Xiaofang $\mathrm{Meng}^{a}$ and Lianglin Xiong ${ }^{b}$ \\ ${ }^{a}$ Department of Mathematics, Yunnan University \\ Kunming, Yunnan 650091 \\ People's Republic of China \\ ${ }^{b}$ School of Mathematics and Computer Science \\ Yunnan Minzu University, Kunming, Yunnan 650500 \\ People's Republic of China
}

\begin{abstract}
In this paper, a class of neutral type high-order Hopfield neural networks with mixed time-varying delays and leakage delays on time scales is proposed. Based on the exponential dichotomy of linear dynamic equations on time scales, Banach's fixed point theorem and the theory of calculus on time scales, some sufficient conditions are obtained for the existence and global exponential stability of pseudo almost periodic solutions for this class of neural networks. Our results are completely new. Finally, we present an example to illustrate our results are effective. Our example also shows that the continuous-time neural network and its discrete-time analogue have the same dynamical behaviors for the pseudo almost periodicity.
\end{abstract}

Key words: Hopfield neural networks; Mixed time-varying delays; Leakage delays; Pseudo almost periodic solutions; Time scales.

\section{Introduction}

Due to the fact that high-order Hopfield neural networks (HHNNs) have stronger approximation property, faster convergence rate, greater storage capacity, and higher fault tolerance than lower-order neural networks, high-order Hopfield neural networks have been the object

\footnotetext{
*This work is supported by the National Natural Sciences Foundation of People's Republic of China under Grants 11361072 and 11461082.

${ }^{\dagger}$ The corresponding author.
} 
of intensive analysis by numerous authors in recent years. In particular, there have been extensive results on the problem of the existence and stability of equilibrium points, periodic solutions and almost periodic solutions of HHNNs in the literature. We refer the reader to [1-9] and the references cited therein. For example, authors of [1] studied the problem of global exponential stability properties of such high-order Hopfield-type neural networks by utilizing Lyapunov functions; authors of [2] derived some sufficient conditions for the global asymptotic stability of equilibrium point of HHNNs with constant time delays in terms of linear matrix inequality.

In fact, it is natural and important that systems will contain some information about the derivative of the past state to further describe and model the dynamics for such complex neural reactions [10], many authors investigated the dynamical behaviors of neutral type neural networks. For example, authors of [11-15] studied the stability, periodic solutions and almost periodic solutions for different classes of neutral type neural networks, respectively.

As we known, time delays inevitably exist in biological and artificial neural networks because of the finite switching speed of neurons and amplifiers [16], which can also affect the stability of neural network systems and may lead to some complex dynamic behaviors such as oscillation, chaos and instability. In reality, the mixed time-varying delays should be taken into account when modeling realistic neural networks [17]. A leakage delay, which is the time delay in the leakage term of the systems and a considerable factor affecting dynamics for the worse in the systems, is being put to use in the problem of stability for neural networks. Such time delays in the leakage term are difficult to handle but has great impact on the dynamical behavior of neural networks [18-21]. Therefore, it is significant to consider neural networks with time delays in leakage terms.

Besides, both continuous-time and discrete-time neural networks have equally importance in various applications and the theory of time scales was initiated by Hilger [22] in his Ph.D. thesis in 1988, which can unify the continuous and discrete cases. Many authors have studied the dynamics of neural networks on time scales [15,23-25]. For example, in paper [24], some sufficient conditions for the existence and global exponential stability of almost automorphic solutions for a class of neutral type HHNNs with delays in leakage terms on time scales are obtained; in paper [25], the existence and global exponential stability of anti-periodic solutions for competitive neural networks with delays in the leakage terms on time scales are investigated.

On the other hand, the concept of pseudo-almost periodicity, which is the central subject in this paper, was introduced by Zhang [26] in the early nineties. As pointed out by Dads et al. in [27], it would be of great interest to study the dynamics of pseudo almost periodic systems with time delays. Pseudo almost periodic solutions in the context of differential equations were studied by several authors in [28-41]. For example, authors of [40] studied the existence and the global exponential stability of the positive pseudo almost periodic solutions, which are more general and complicated than periodic and almost periodic solutions; authors of [41] studied the existence and uniqueness of pseudo almost periodic solution of the shunting inhibitory cellular neural networks with time-varying delays in the leakage terms by using the exponential dichotomy theory and contraction mapping fixed point theorem. Recently, the concept of the pseudo almost periodic function on time scales has been introduced by 
$\mathrm{Li}$ and Wang [42]. However, to the best of our knowledge, there is no paper published on the existence and stability of pseudo almost periodic solutions for neutral type HHNNs with mixed time-varying delays and leakage delays on time scales, which is very important in theories and applications and also is a very challenging problem.

Motivated by the above discussion, in this paper, we propose a neutral type high-order Hopfield neural network with mixed time-varying delays and leakage delays on time scales as follows:

$$
\begin{aligned}
x_{i}^{\nabla}(t)= & -c_{i}(t) x_{i}\left(t-\delta_{i}(t)\right)+\sum_{j=1}^{n} a_{i j}(t) f_{j}\left(x_{j}(t)\right) \\
& +\sum_{j=1}^{n} b_{i j}(t) g_{j}\left(x_{j}\left(t-\tau_{i j}(t)\right)\right)+\sum_{j=1}^{n} d_{i j}(t) \int_{t-\sigma_{i j}(t)}^{t} h_{j}\left(x_{j}^{\nabla}(s)\right) \nabla s \\
& +\sum_{j=1}^{n} \sum_{l=1}^{n} T_{i j l}(t) k_{j}\left(x_{j}\left(t-\xi_{i j l}(t)\right)\right) k_{l}\left(x_{l}\left(t-\zeta_{i j l}(t)\right)\right)+I_{i}(t), t \in \mathbb{T},
\end{aligned}
$$

where $\mathbb{T}$ is an almost periodic time scale, $i=1,2, \ldots, n, n$ corresponds to the number of units in a neural network; $x_{i}(t)$ denotes the activation of the $i$ th neuron at time $t ; c_{i}(t)>0$ represents the rate with the $i$ th unit will reset its potential to the resting state in isolation when disconnected from the network and external inputs at time $t ; a_{i j}(t), b_{i j}(t)$ and $d_{i j}(t)$ represent the delayed strengths of connectivity and neutral delayed strengths of connectivity between cell $i$ and $j$ at time $t$, respectively; $T_{i j l}(t)$ denotes the second-order connection weights of the neural network; $f_{j}, g_{j}, h_{j}$ and $k_{j}$ are the activation functions in system (1.1); $I_{i}(t)$ is an external input on the $i$ th unit at time $t ; \delta_{i}$ denotes the leakage delay at time $t$ and satisfy $t-\delta_{i}(t) \in \mathbb{T}, \tau_{i j}, \sigma_{i j}, \xi_{i j l}$ and $\zeta_{i j l}$ are transmission delays at time $t$ and satisfy $t-\tau_{i j}(t) \in \mathbb{T}$, $t-\sigma_{i j}(t) \in \mathbb{T}, t-\xi_{i j l}(t) \in \mathbb{T}, t-\zeta_{i j l}(t) \in \mathbb{T}$ for $t \in \mathbb{T}$.

For convenience, we let $[a, b]_{\mathbb{T}}=\{t \mid t \in[a, b] \cap \mathbb{T}\}$. And we introduce the following notations:

$$
\begin{gathered}
c_{i}^{+}=\sup _{t \in \mathbb{T}}\left|c_{i}(t)\right|, \quad c_{i}^{-}=\inf _{t \in \mathbb{T}}\left|c_{i}(t)\right|, \quad \delta_{i}^{+}=\sup _{t \in \mathbb{T}}\left|\delta_{i}(t)\right|, \quad \tau_{i j}^{+}=\sup _{t \in \mathbb{T}}\left|\tau_{i j}(t)\right|, \\
\sigma_{i j}^{+}=\sup _{t \in \mathbb{T}}\left|\sigma_{i j}(t)\right|, \quad \xi_{i j l}^{+}=\sup _{t \in \mathbb{T}}\left|\xi_{i j l}(t)\right|, \quad \zeta_{i j l}^{+}=\sup _{t \in \mathbb{T}}\left|\zeta_{i j l}(t)\right|, \quad a_{i j}^{+}=\sup _{t \in \mathbb{T}}\left|a_{i j}(t)\right|, \\
b_{i j}^{+}=\sup _{t \in \mathbb{T}}\left|b_{i j}(t)\right|, \quad d_{i j}^{+}=\sup _{t \in \mathbb{T}}\left|d_{i j}(t)\right|, \quad T_{i j l}^{+}=\sup _{t \in \mathbb{T}}\left|T_{i j l}(t)\right|, \quad i, j, l=1,2, \ldots, n .
\end{gathered}
$$

The initial condition associated with system (1.1) is of the form

$$
x_{i}(s)=\varphi_{i}(s), x_{i}^{\nabla}(s)=\varphi_{i}^{\nabla}(s), s \in[-\theta, 0]_{\mathbb{T}},
$$

where $\theta=\max \{\delta, \tau, \sigma, \xi, \zeta\}, \delta=\max _{1 \leq i \leq n}\left\{\delta_{i}^{+}\right\}, \tau=\max _{1 \leq i, j \leq n}\left\{\tau_{i j}^{+}\right\}, \sigma=\max _{1 \leq i, j \leq n}\left\{\sigma_{i j}^{+}\right\}, \xi=\max _{1 \leq i, j, l \leq n}\left\{\xi_{i j l}^{+}\right\}$, $\zeta=\max _{1 \leq i, j, l \leq n}\left\{\zeta_{i j l}^{+}\right\}, i, j, l=1,2, \ldots, n, \varphi_{k}(\cdot)$ denotes a real-valued bounded $\nabla$-differentiable function defined on $[-\theta, 0]_{\mathbb{T}}$. 
This paper is organized as follows. In Section 2, we introduce some definitions, make some preparations for later sections and extend the almost periodic theory on time scales with the delta derivative to that with the nabla derivative. In Section 3, by utilizing Banach's fixed point theorem and the theory of calculus on time scales, we present some sufficient conditions for the existence of pseudo almost periodic solutions of (1.1). In Section 4, we prove that the pseudo almost periodic solution obtained in Section 3 is globally exponentially stable. In Section 5, we give an example to demonstrate the feasibility of our results.

\section{Preliminaries}

In this section, we shall first recall some fundamental definitions and lemmas. Also, we extend the almost periodic theory on time scales with the delta derivative to that with the nabla derivative.

A time scale $\mathbb{T}$ is an arbitrary nonempty closed subset of the real set $\mathbb{R}$ with the topology and ordering inherited from $\mathbb{R}$. The forward jump operator $\sigma: \mathbb{T} \rightarrow \mathbb{T}$ is defined by $\sigma(t)=$ $\inf \{s \in \mathbb{T}, s>t\}$ for all $t \in \mathbb{T}$, while the backward jump operator $\rho: \mathbb{T} \rightarrow \mathbb{T}$ is defined by $\rho(t)=\sup \{s \in \mathbb{T}, s<t\}$ for all $t \in \mathbb{T}$.

A point $t \in \mathbb{T}$ is called left-dense if $t>\inf \mathbb{T}$ and $\rho(t)=t$, left-scattered if $\rho(t)<t$, right-dense if $t<\sup \mathbb{T}$ and $\sigma(t)=t$, and right-scattered if $\sigma(t)>t$. If $\mathbb{T}$ has a left-scattered maximum $m$, then $\mathbb{T}^{k}=\mathbb{T} \backslash\{m\}$; otherwise $\mathbb{T}^{k}=\mathbb{T}$. If $\mathbb{T}$ has a right-scattered minimum $m$, then $\mathbb{T}_{k}=\mathbb{T} \backslash\{m\}$; otherwise $\mathbb{T}_{k}=\mathbb{T}$. Finally, the backwards graininess function $\nu: \mathbb{T}_{k} \rightarrow[0, \infty)$ is defined by $\nu(t)=t-\rho(t)$.

A function $f: \mathbb{T} \rightarrow \mathbb{R}$ is ld-continuous provided it is continuous at left-dense point in $\mathbb{T}$ and its right-side limits exist at right-dense points in $\mathbb{T}$.

Definition 2.1. (43, 44) Let $f: \mathbb{T} \rightarrow \mathbb{R}$ be a function and $t \in \mathbb{T}_{k}$. Then we define $f^{\nabla}(t)$ to be the number (provided its exists) with the property that given any $\varepsilon>0$, there is a neighborhood $U$ of $t$ (i.e, $U=(t-\delta, t+\delta) \cap \mathbb{T}$ for some $\delta>0)$ such that

$$
\left|f(\rho(t))-f(s)-f^{\nabla}(t)(\rho(t)-s)\right| \leq \varepsilon|\rho(t)-s|
$$

for all $s \in U$, we call $f^{\nabla}(t)$ the nabla derivative of $f$ at $t$.

Let $f: \mathbb{T} \rightarrow \mathbb{R}$ be ld-continuous. If $F^{\nabla}(t)=f(t)$, then we define the nabla integral by $\int_{a}^{b} f(t) \nabla t=F(b)-F(a)$.

A function $p: \mathbb{T} \rightarrow \mathbb{R}$ is called $\nu$-regressive if $1-\nu(t) p(t) \neq 0$ for all $t \in \mathbb{T}_{k}$. The set of all $\nu$-regressive and left-dense continuous functions $p: \mathbb{T} \rightarrow \mathbb{R}$ will be denoted by $\mathcal{R}_{\nu}=\mathcal{R}_{\nu}(\mathbb{T})=$ $\mathcal{R}_{\nu}(\mathbb{T}, \mathbb{R})$. We define the set $\mathcal{R}_{\nu}^{+}=\mathcal{R}_{\nu}^{+}(\mathbb{T}, \mathbb{R})=\left\{p \in \mathcal{R}_{\nu}: 1-\nu(t) p(t)>0, \forall t \in \mathbb{T}\right\}$.

If $p \in \mathcal{R}_{\nu}$, then we define the nabla exponential function by

$$
\hat{e}_{p}(t, s)=\exp \left(\int_{s}^{t} \hat{\xi}_{\nu(\tau)}(p(\tau)) \nabla \tau\right), \text { for } t, s \in \mathbb{T}
$$

with the $\nu$-cylinder transformation

$$
\hat{\xi}_{h}(z)= \begin{cases}-\frac{\log (1-h z)}{h} & \text { if } h \neq 0 \\ z & \text { if } h=0\end{cases}
$$


Let $p, q \in \mathcal{R}_{\nu}$, then we define a circle plus addition by $\left(p \oplus_{\nu} q\right)(t)=p(t)+q(t)-\nu(t) p(t) q(t)$, for all $t \in \mathbb{T}_{k}$. For $p \in \mathcal{R}_{\nu}$, define a circle minus $p$ by $\ominus_{\nu} p=-\frac{p}{1-\nu p}$.

Lemma 2.1. (43, 44) Let $p, q \in \mathcal{R}_{\nu}$, and $s, t, r \in \mathbb{T}$. Then

(i) $\hat{e}_{0}(t, s) \equiv 1$ and $\hat{e}_{p}(t, t) \equiv 1$;

(ii) $\hat{e}_{p}(\rho(t), s)=(1-\nu(t) p(t)) \hat{e}_{p}(t, s)$;

(iii) $\hat{e}_{p}(t, s)=\frac{1}{\hat{e}_{p}(s, t)}=\hat{e}_{\ominus \nu}(s, t)$;

(iv) $\hat{e}_{p}(t, s) \hat{e}_{p}(s, r)=\hat{e}_{p}(t, r)$;

(v) $\left(\hat{e}_{p}(t, s)\right)^{\nabla}=p(t) \hat{e}_{p}(t, s)$.

Lemma 2.2. (43, 44]) Let $f, g$ be nabla differentiable functions on $\mathbb{T}$, then

(i) $\left(v_{1} f+v_{2} g\right)^{\nabla}=v_{1} f^{\nabla}+v_{2} g^{\nabla}$, for any constants $v_{1}, v_{2}$;

(ii) $(f g)^{\nabla}(t)=f^{\nabla}(t) g(t)+f(\rho(t)) g^{\nabla}(t)=f(t) g^{\nabla}(t)+f^{\nabla}(t) g(\rho(t))$;

(iii) If $f$ and $f^{\nabla}$ are continuous, then $\left(\int_{a}^{t} f(t, s) \nabla s\right)^{\nabla}=f(\rho(t), t)+\int_{a}^{t} f(t, s) \nabla s$.

Lemma 2.3. (43, 44) Assume $p \in \mathcal{R}_{\nu}$ and $t_{0} \in \mathbb{T}$. If $1-\nu(t) p(t)>0$ for $t \in \mathbb{T}$, then $\hat{e}_{p}\left(t, t_{0}\right)>0$ for all $t \in \mathbb{T}$.

Lemma 2.4. Suppose that $f(t)$ is an ld-continuous function and $c(t)$ is a positive ld-continuous function which satisfies that $c(t) \in \mathcal{R}_{\nu}^{+}$. Let

$$
g(t)=\int_{t_{0}}^{t} \hat{e}_{-c}(t, \rho(s)) f(s) \nabla s,
$$

where $t_{0} \in \mathbb{T}$, then

$$
g^{\nabla}(t)=f(t)-c(t) \int_{t_{0}}^{t} \hat{e}_{-c}(t, \rho(s)) f(s) \nabla s
$$

Proof.

$$
\begin{aligned}
g^{\nabla}(t) & =\left(\int_{t_{0}}^{t} \hat{e}_{-c}(t, \rho(s)) f(s) \nabla s\right)^{\nabla} \\
& =\left(\hat{e}_{-c}\left(t, t_{0}\right) \int_{t_{0}}^{t} \hat{e}_{-c}\left(t_{0}, \rho(s)\right) f(s) \nabla s\right)^{\nabla} \\
& =\hat{e}_{-c}\left(\rho(t), t_{0}\right) \hat{e}_{-c}\left(t_{0}, \rho(t)\right) f(t)-c(t) \hat{e}_{-c}\left(t, t_{0}\right) \int_{t_{0}}^{t} \hat{e}_{-c}\left(t_{0}, \rho(s)\right) f(s) \nabla s \\
& =f(t)-c(t) \int_{t_{0}}^{t} \hat{e}_{-c}(t, \rho(s)) f(s) \nabla s
\end{aligned}
$$


Definition 2.2. $(45,46])$ A time scale $\mathbb{T}$ is called an almost periodic time scale if

$$
\Pi:=\{\tau \in \mathbb{R}: t \pm \tau \in \mathbb{T}, \forall t \in \mathbb{T}\} \neq\{0\} .
$$

Definition 2.3. (45, 46]) Let $\mathbb{T}$ be an almost periodic time scale. A function $f \in C\left(\mathbb{T}, \mathbb{R}^{n}\right)$ is called an almost periodic on $\mathbb{T}$, if for any $\varepsilon>0$, the set

$$
E(\varepsilon, f)=\{\tau \in \Pi:|f(t+\tau)-f(t)|<\varepsilon, \forall t \in \mathbb{T}\}
$$

is a relatively dense in $\mathbb{T}$; that is, for any given $\varepsilon>0$, there exists a constant $l(\varepsilon)>0$ such that each interval of length $l(\varepsilon)$ contains at least one $\tau=\tau(\varepsilon) \in E(\varepsilon, f)$ such that

$$
|f(t+\tau)-f(t)|<\varepsilon, \forall t \in \mathbb{T} .
$$

The set $E(\varepsilon, f)$ is called the $\varepsilon$-translation set of $f(t), \tau$ is called the $\varepsilon$-translation number of $f(t)$ and $l(\varepsilon)$ is called the contain interval length of $E(\varepsilon, f)$.

Let $A P(\mathbb{T})=\left\{f \in C\left(\mathbb{T}, \mathbb{R}^{n}\right): f\right.$ is almost periodic $\}$ and $B C\left(\mathbb{T}, \mathbb{R}^{n}\right)$ denote the space of all bounded continuous functions from $\mathbb{T}$ to $\mathbb{R}^{n}$. Define the class of functions $P A P_{0}(\mathbb{T})$ as follows:

$$
\begin{aligned}
\operatorname{PAP}_{0}(\mathbb{T})= & \left\{f \in B C\left(\mathbb{T}, \mathbb{R}^{n}\right): f \text { is } \nabla\right. \text { - measurable such that } \\
& \left.\lim _{r \rightarrow+\infty} \frac{1}{2 r} \int_{t_{0}-r}^{t_{0}+r}|f(s)| \nabla s=0 \text {, where } t_{0} \in \mathbb{T}, r \in \Lambda\right\} .
\end{aligned}
$$

Similar to Definition 4.1 in [42], we give

Definition 2.4. A function $f \in C\left(\mathbb{T}, \mathbb{R}^{n}\right)$ is called pseudo almost periodic if $f=g+\phi$, where $g \in A P(\mathbb{T})$ and $\phi \in P A P_{0}(\mathbb{T})$. Denote by $P A P(\mathbb{T})$ the set of pseudo almost periodic functions.

By Definition 2.4, one can easily show that

Lemma 2.5. If $f, g \in P A P(\mathbb{T})$, then $f+g, f g \in P A P(\mathbb{T})$; if $f \in P A P(\mathbb{T}), g \in A P(\mathbb{T})$, then $f g \in P A P(\mathbb{T})$.

Lemma 2.6. If $f \in C(\mathbb{R}, \mathbb{R})$ satisfies the Lipschitcz condition, $\varphi \in P A P(\mathbb{T})$ and $\theta \in C(\mathbb{T}, \Lambda)$, then $f(\varphi(t-\theta(t))) \in P A P(\mathbb{T})$.

Proof. From Definition 2.4, we have $\varphi=\varphi_{1}+\varphi_{2}$, where $\varphi_{1} \in A P(\mathbb{T})$ and $\varphi_{2} \in P A P_{0}(\mathbb{T})$. Set

$$
\begin{aligned}
E(t) & =f(\varphi(t-\theta(t)))=f\left(\varphi_{1}(t-\theta(t))\right)+\left[f\left(\varphi_{1}(t-\theta(t))+\varphi_{2}(t-\theta(t))\right)-f\left(\varphi_{1}(t-\theta(t))\right)\right] \\
& :=E_{1}(t)+E_{2}(t) .
\end{aligned}
$$

Firstly, it follows from Theorem 2.11 in [45] that $E_{1} \in A P(\mathbb{T})$. Then, we show that $E_{2} \in$ $P A P_{0}(\mathbb{T})$ because

$$
\lim _{r \rightarrow+\infty} \frac{1}{2 r} \int_{t_{0}-r}^{t_{0}+r}\left|E_{2}(s)\right| \nabla s
$$




$$
\begin{aligned}
& =\lim _{r \rightarrow+\infty} \frac{1}{2 r} \int_{t_{0}-r}^{t_{0}+r}\left|f\left(\varphi_{1}(s-\theta(s))+\varphi_{2}(s-\theta(s))\right)-f\left(\varphi_{1}(s-\theta(s))\right)\right| \nabla s \\
& \leq \lim _{r \rightarrow+\infty} \frac{L}{2 r} \int_{t_{0}-r}^{t_{0}+r}\left|\varphi_{2}(s-\theta(s))\right| \nabla s=0 .
\end{aligned}
$$

Thus $E_{2} \in P A P_{0}(\mathbb{T})$. So $E \in P A P(\mathbb{T})$. The proof is complete.

Similar to Definition 2.12 in [45], we give

Definition 2.5. Let $A(t)$ be an $n \times n$ matrix-valued function on $\mathbb{T}$. Then the linear system

$$
x^{\nabla}(t)=A(t) x(t), t \in \mathbb{T}
$$

is said to admit an exponential dichotomy on $\mathbb{T}$ if there exist positive constant $K$, $\alpha$, projection $P$ and the fundamental solution matrix $X(t)$ of (2.1), satisfying

$$
\begin{array}{r}
\left\|X(t) P X^{-1}(s)\right\|_{0} \leq K \hat{e}_{\ominus_{\nu} \alpha}(t, s), s, t \in \mathbb{T}, t \geq s \\
\left\|X(t)(I-P) X^{-1}(s)\right\|_{0} \leq K \hat{e}_{\ominus_{\nu} \alpha}(s, t), s, t \in \mathbb{T}, t \leq s,
\end{array}
$$

where $\|\cdot\|_{0}$ is a matrix norm on $\mathbb{T}$ (say, for example, if $A=\left(a_{i j}\right)_{n \times m}$, then we can take $\left.\|A\|_{0}=\left(\sum_{i=1}^{n} \sum_{j=1}^{m}\left|a_{i j}\right|^{2}\right)^{\frac{1}{2}}\right)$.

Consider the following almost periodic system

$$
x^{\nabla}(t)=A(t) x(t)+f(t), t \in \mathbb{T},
$$

where $A(t)$ is an almost periodic matrix function, $f(t)$ is an almost periodic vector function. Similar to the proof of Theorem 5.2 in [42], we can get the following lemma.

Lemma 2.7. Suppose that $A(t)$ is almost periodic, (2.1) admits an exponential dichotomy and the function $f \in P A P(\mathbb{T})$. Then (2.2) has a unique bounded solution $x \in P A P(\mathbb{T})$ that can be expressed as follows:

$$
x(t)=\int_{-\infty}^{t} X(t) P X^{-1}(\rho(s)) f(s) \nabla s-\int_{t}^{+\infty} X(t)(I-P) X^{-1}(\rho(s)) f(s) \nabla s,
$$

where $X(t)$ is the fundamental solution matrix of (2.1).

Similar to the proof of Lemma 2.15 in [45], we have

Lemma 2.8. Let $c_{i}(t): \mathbb{T} \rightarrow \mathbb{R}^{+}$be an almost periodic function, $-c_{i} \in \mathcal{R}_{\nu}^{+}, \mathbb{T} \in \Lambda$ and

$$
m\left(c_{i}\right)=\lim _{T \rightarrow \infty} \frac{1}{T} \int_{t}^{t+T} c_{i}(s) \nabla s>0, \quad i=1,2, \ldots, n .
$$

Then the linear system

$$
x^{\nabla}(t)=\operatorname{diag}\left(-c_{1}(t),-c_{2}(t), \ldots,-c_{n}(t)\right) x(t)
$$

admits an exponential dichotomy on $\mathbb{T}$, where $m\left(c_{i}\right)$ denote the mean-value of $c_{i}, i=1,2, \ldots, n$. 


\section{The existence of pseudo almost periodic solution}

In this section, we will state and prove the sufficient conditions for the existence of pseudo almost periodic solutions of (1.1).

Let

$$
\mathbb{B}=\left\{\varphi(t)=\left(\varphi_{1}(t), \varphi_{2}(t), \ldots, \varphi_{n}(t)\right)^{T}: \varphi_{i}(t), \varphi_{i}^{\nabla}(t) \in P A P(\mathbb{T}), i=1,2, \ldots, n\right\}
$$

with the norm $\|\varphi\|_{\mathbb{B}}=\sup _{t \in \mathbb{T}}\|\varphi(t)\|$, where $\|\varphi(t)\|=\max _{1 \leq i \leq n}\left\{\left|\varphi_{i}(t)\right|,\left|\varphi_{i}^{\nabla}(t)\right|\right\}$, then $\mathbb{B}$ is a Banach space.

Throughout this paper, we assume that the following conditions hold:

$\left(H_{1}\right) c_{i} \in C\left(\mathbb{T}, \mathbb{R}^{+}\right)$with $-c_{i} \in \mathcal{R}_{\nu}^{+}$, where $\mathcal{R}_{\nu}^{+}$denotes the set of positively regressive functions from $\mathbb{T}$ to $\mathbb{R}, i=1,2, \ldots, n$;

$\left(H_{2}\right) a_{i j}, b_{i j}, d_{i j}, T_{i j l} \in A P(\mathbb{T}), \delta_{i}, \tau_{i j}, \sigma_{i j}, \xi_{i j l}, \zeta_{i j l} \in C(\mathbb{T}, \Lambda)$ and $I_{i} \in P A P(\mathbb{T})$;

$\left(H_{3}\right)$ Functions $f_{j}, g_{j}, h_{j}, k_{j} \in C(\mathbb{R}, \mathbb{R})$ and there exist positive constants $L_{j}^{f}, L_{j}^{g}, L_{j}^{h}, L_{j}^{k}$ such that

$$
\begin{aligned}
& \left|f_{j}(u)-f_{j}(v)\right| \leq L_{j}^{f}|u-v|,\left|g_{j}(u)-g_{j}(v)\right| \leq L_{j}^{g}|u-v|, \\
& \left|h_{j}(u)-h_{j}(v)\right| \leq L_{j}^{h}|u-v|,\left|k_{j}(u)-k_{j}(v)\right| \leq L_{j}^{k}|u-v|,
\end{aligned}
$$

where $u, v \in \mathbb{R}$ and $f_{j}(0)=g_{j}(0)=h_{j}(0)=k_{j}(0)=0, j=1,2, \ldots, n$.

Theorem 3.1. Let $\left(H_{1}\right)-\left(H_{3}\right)$ hold. Suppose that

$\left(H_{4}\right)$ there exists a positive constant $r$ such that

$$
\begin{aligned}
& \max _{1 \leq i \leq n}\left\{\frac{\rho_{i}}{c_{i}^{-}}, \frac{c_{i}^{+}+c_{i}^{-}}{c_{i}^{-}} \rho_{i}\right\}+\max \left\{K_{1}, K_{2}\right\} \leq r, \\
& 0<\frac{\rho_{i}}{r}<\frac{c_{i}^{-}}{c_{i}^{+}+c_{i}^{-}}<c_{i}^{-}, \quad i=1,2, \ldots, n,
\end{aligned}
$$

where

$$
\begin{aligned}
\rho_{i}= & \left(c_{i}^{+} \delta_{i}^{+}+\sum_{j=1}^{n} a_{i j}^{+} L_{j}^{f}+\sum_{j=1}^{n} b_{i j}^{+} L_{j}^{g}+\sum_{j=1}^{n} d_{i j}^{+} \sigma_{i j}^{+} L_{j}^{h}\right. \\
& \left.+\sum_{j=1}^{n} \sum_{l=1}^{n} T_{i j l}^{+} L_{j}^{k} L_{l}^{k}\right) r, \quad i=1,2, \ldots, n, \\
K_{1}= & \max _{1 \leq i \leq n}\left\{\frac{I_{i}^{+}}{c_{i}^{-}}\right\}, \quad K_{2}=\max _{1 \leq i \leq n}\left\{\frac{c_{i}^{+}+c_{i}^{-}}{c_{i}^{-}} I_{i}^{+}\right\} .
\end{aligned}
$$

Then system (1.1) has at least one pseudo almost periodic solution in the region $\mathbb{E}=\{\varphi \in$ $\left.\mathbb{B}:\|\varphi\|_{\mathbb{B}} \leq r\right\}$. 
Proof. Rewrite (1.1) in the form

$$
\begin{aligned}
x_{i}^{\nabla}(t)= & -c_{i}(t) x_{i}(t)+c_{i}(t) \int_{t-\delta_{i}(t)}^{t} x_{i}^{\nabla}(s) \nabla s+\sum_{j=1}^{n} a_{i j}(t) f_{j}\left(x_{j}(t)\right) \\
& +\sum_{j=1}^{n} b_{i j}(t) g_{j}\left(x_{j}\left(t-\tau_{i j}(t)\right)\right)+\sum_{j=1}^{n} d_{i j}(t) \int_{t-\sigma_{i j}(t)}^{t} h_{j}\left(x_{j}^{\nabla}(s)\right) \nabla s \\
& +\sum_{j=1}^{n} \sum_{l=1}^{n} T_{i j l}(t) k_{j}\left(x_{j}\left(t-\xi_{i j l}(t)\right)\right) k_{l}\left(x_{l}\left(t-\zeta_{i j l}(t)\right)\right)+I_{i}(t), \quad t \in \mathbb{T}, \quad i=1,2, \ldots, n .
\end{aligned}
$$

For any $\varphi \in \mathbb{B}$, we consider the following system

$$
x_{i}^{\nabla}(t)=-c_{i}(t) x_{i}(t)+F_{i}(t, \varphi)+I_{i}(t), \quad t \in \mathbb{T}, \quad i=1,2, \ldots, n,
$$

where

$$
\begin{aligned}
F_{i}(t, \varphi)= & c_{i}(t) \int_{t-\delta_{i}(t)}^{t} \varphi_{i}^{\nabla}(s) \nabla s+\sum_{j=1}^{n} a_{i j}(t) f_{j}\left(\varphi_{j}(t)\right) \\
& +\sum_{j=1}^{n} b_{i j}(t) g_{j}\left(\varphi_{j}\left(t-\tau_{i j}(t)\right)\right)+\sum_{j=1}^{n} d_{i j}(t) \int_{t-\sigma_{i j}(t)}^{t} h_{j}\left(\varphi_{j}^{\nabla}(s)\right) \nabla s \\
& +\sum_{j=1}^{n} \sum_{l=1}^{n} T_{i j l}(t) k_{j}\left(\varphi_{j}\left(t-\xi_{i j l}(t)\right)\right) k_{l}\left(\varphi_{l}\left(t-\zeta_{i j l}(t)\right)\right) .
\end{aligned}
$$

Since $\min _{1 \leq i \leq n}\left\{\inf _{t \in \mathbb{T}} c_{i}(t)\right\}>0$, it follows from Lemma 2.8 that the linear system

$$
x_{i}^{\nabla}(t)=-c_{i}(t) x_{i}(t), i=1,2, \ldots, n
$$

admits an exponential dichotomy on $\mathbb{T}$. Thus, by Lemma 2.7, we know that system (3.1) has exactly one pseudo almost periodic solution which can be expressed as follows:

$$
x_{\varphi}=\left(x_{\varphi_{1}}, x_{\varphi_{2}}, \ldots, x_{\varphi_{n}}\right)^{T},
$$

where

$$
x_{\varphi_{i}}(t)=\int_{-\infty}^{t} \hat{e}_{-c_{i}}(t, \rho(s))\left(F_{i}(s, \varphi)+I_{i}(s)\right) \nabla s, i=1,2, \ldots, n .
$$

Define the following operator

$$
\begin{aligned}
\Phi: \mathbb{E} & \rightarrow \mathbb{E} \\
\left(\varphi_{1}, \varphi_{2}, \ldots, \varphi_{n}\right)^{T} & \rightarrow\left(x_{\varphi_{1}}, x_{\varphi_{2}}, \ldots, x_{\varphi_{n}}\right)^{T}
\end{aligned}
$$

We will show that $\Phi$ is a contraction. 
First, we show that for any $\varphi \in \mathbb{E}$, we have $\Phi \varphi \in \mathbb{E}$. Note that

$$
\begin{aligned}
\left|F_{i}(s, \varphi)\right|= & \mid c_{i}(s) \int_{s-\delta_{i}(s)}^{s} \varphi_{i}^{\nabla}(u) \nabla u+\sum_{j=1}^{n} a_{i j}(s) f_{j}\left(\varphi_{j}(s)\right) \\
& +\sum_{j=1}^{n} b_{i j}(s) g_{j}\left(\varphi_{j}\left(s-\tau_{i j}(s)\right)\right)+\sum_{j=1}^{n} d_{i j}(s) \int_{s-\sigma_{i j}(s)}^{s} h_{j}\left(\varphi_{j}^{\nabla}(u)\right) \nabla u \\
& +\sum_{j=1}^{n} \sum_{l=1}^{n} T_{i j l}(s) k_{j}\left(\varphi_{j}\left(s-\xi_{i j l}(s)\right)\right) k_{l}\left(\varphi_{l}\left(s-\zeta_{i j l}(s)\right)\right) \mid \\
\leq & c_{i}^{+}\left|\int_{s-\delta_{i}(s)}^{s} \varphi_{i}^{\nabla}(u) \nabla u\right|+\sum_{j=1}^{n} a_{i j}^{+}\left|f_{j}\left(\varphi_{j}(s)\right)\right| \\
& +\sum_{j=1}^{n} b_{i j}^{+}\left|g_{j}\left(\varphi_{j}\left(s-\tau_{i j}(s)\right)\right)\right|+\sum_{j=1}^{n} d_{i j}^{+}\left|\int_{s-\sigma_{i j}(s)}^{s} h_{j}\left(\varphi_{j}^{\nabla}(u)\right) \nabla u\right| \\
& +\sum_{j=1}^{n} \sum_{l=1}^{n} T_{i j l}^{+}\left|k_{j}\left(\varphi_{j}\left(s-\xi_{i j l}(s)\right)\right) k_{l}\left(\varphi_{l}\left(s-\zeta_{i j l}(s)\right)\right)\right| \\
\leq & c_{i}^{+} \delta_{i}^{+}\left|\varphi_{i}^{\nabla}(s)\right|+\sum_{j=1}^{n} a_{i j}^{+} L_{j}^{f}\left|\varphi_{j}(s)\right|+\sum_{j=1}^{n} b_{i j}^{+} L_{j}^{g}\left|\varphi_{j}\left(s-\tau_{i j}(s)\right)\right| \\
& +\sum_{j=1}^{n} d_{i j}^{+} \sigma_{i j}^{+} L_{j}^{h}\left|\varphi_{j}^{\nabla}(s)\right|+\sum_{j=1}^{n} \sum_{l=1}^{n} T_{i j l}^{+} L_{j}^{k} L_{l}^{k}\left|\varphi_{j}\left(s-\xi_{i j l}(s)\right)\right|\left|\varphi_{l}\left(s-\zeta_{i j l}(s)\right)\right| \\
\leq & \left.\left(c_{i}^{+} \delta_{i}^{+}+\sum_{j=1}^{n} a_{i j}^{+} L_{j}^{f}+\sum_{j=1}^{n} b_{i j}^{+} L_{j}^{g}+\sum_{j=1}^{n} d_{i j}^{+} \sigma_{i j}^{+} L_{j}^{h}+\sum_{j=1}^{n} \sum_{l=1}^{n} T_{i j l}^{+} L_{j}^{k} L_{l}^{k}\right)\|\varphi\| \|_{\mathbb{B}}^{n}{ }^{n} T_{i j l}^{+} L_{j}^{k} L_{l}^{k}\right) r \\
\leq & \left(c_{i}^{+} \delta_{i}^{+}+\sum_{j=1}^{n} a_{i j}^{+} L_{j}^{f}+\sum_{j=1}^{n} b_{i j}^{+} L_{j}^{g}+\sum_{j=1}^{n} d_{i j}^{+} \sigma_{i j}^{+} L_{j}^{h}+\sum_{j=1}^{n} \sum_{l=1}\right. \\
= & \rho_{i}, i=1,2, \ldots, n .
\end{aligned}
$$

Therefore, we can get

$$
\begin{aligned}
\sup _{t \in \mathbb{T}}\left|x_{\varphi_{i}}(t)\right| & =\sup _{t \in \mathbb{T}}\left|\int_{-\infty}^{t} \hat{e}_{-c_{i}}(t, \rho(s))\left(F_{i}(s, \varphi)+I_{i}(s)\right) \nabla s\right| \\
& \leq \sup _{t \in \mathbb{T}} \int_{-\infty}^{t} \hat{e}_{-c_{i}^{-}}(t, \rho(s))\left|F_{i}(s, \varphi)\right| \nabla s+\frac{I_{i}^{+}}{c_{i}^{-}} \\
& \leq \frac{\rho_{i}}{c_{i}^{-}}+\frac{I_{i}^{+}}{c_{i}^{-}} \leq r, \quad i=1,2, \ldots, n .
\end{aligned}
$$

On the other hand, for $i=1,2, \ldots, n$, we have

$$
\sup _{t \in \mathbb{T}}\left|x_{\varphi_{i}}^{\nabla}(t)\right|=\sup _{t \in \mathbb{T}}\left|F_{i}(t, \varphi)+I_{i}(t)-c_{i}(t) \int_{-\infty}^{t} \hat{e}_{-c_{i}}(t, \rho(s))\left(F_{i}(s, \varphi)+I_{i}(s)\right) \nabla s\right|
$$




$$
\begin{aligned}
\leq & \left(c_{i}^{+} \delta_{i}^{+}+\sum_{j=1}^{n} a_{i j}^{+} L_{j}^{f}+\sum_{j=1}^{n} b_{i j}^{+} L_{j}^{g}+\sum_{j=1}^{n} d_{i j}^{+} \sigma_{i j}^{+} L_{j}^{h}+\sum_{j=1}^{n} \sum_{l=1}^{n} T_{i j l}^{+} L_{j}^{k} L_{l}^{k}\right) r+I_{i}^{+} \\
& +c_{i}^{+} \int_{-\infty}^{t} \hat{e}_{-c_{i}^{-}}(t, \rho(s))\left(c_{i}^{+} \delta_{i}^{+}+\sum_{j=1}^{n} a_{i j}^{+} L_{j}^{f}+\sum_{j=1}^{n} b_{i j}^{+} L_{j}^{g}+\sum_{j=1}^{n} d_{i j}^{+} \sigma_{i j}^{+} L_{j}^{h}\right. \\
& \left.+\sum_{j=1}^{n} \sum_{l=1}^{n} T_{i j l}^{+} L_{j}^{k} L_{l}^{k}\right) r \nabla s+\frac{I_{i}^{+}}{c_{i}^{-}} \\
\leq & \frac{c_{i}^{+}+c_{i}^{-}}{c_{i}^{-}} \rho_{i}+\frac{c_{i}^{+}+c_{i}^{-}}{c_{i}^{-}} I_{i}^{+} \leq r .
\end{aligned}
$$

In view of $\left(H_{4}\right)$, we have

$$
\|\Phi(\varphi)\|_{\mathbb{B}}=\max _{1 \leq i \leq n}\left\{\sup _{t \in \mathbb{T}}\left|x_{\varphi_{i}}(t)\right|, \sup _{t \in \mathbb{T}}\left|x_{\varphi_{i}}^{\nabla}(t)\right|\right\} \leq r,
$$

which implies that $\Phi \varphi \in \mathbb{E}$. Hence, the mapping $\Phi$ is a self-mapping from $\mathbb{E}$ to $\mathbb{E}$. Next, we shall prove that $\Phi$ is a contraction mapping. For any $\varphi, \psi \in \mathbb{E}$, we denote

$$
\begin{aligned}
H_{i}(s, \varphi, \psi)= & c_{i}(s) \int_{s-\delta_{i}(s)}^{s}\left[\varphi_{i}^{\nabla}(u)-\psi_{i}^{\nabla}(u)\right] \nabla u+\sum_{j=1}^{n} a_{i j}(s)\left[f_{j}\left(\varphi_{j}(s)\right)-f_{j}\left(\psi_{j}(s)\right)\right] \\
& +\sum_{j=1}^{n} b_{i j}(s)\left[g_{j}\left(\varphi_{j}\left(s-\tau_{i j}(s)\right)\right)-g_{j}\left(\psi_{j}\left(s-\tau_{i j}(s)\right)\right)\right] \\
& +\sum_{j=1}^{n} d_{i j}(s) \int_{s-\sigma_{i j}(s)}^{s}\left[h_{j}\left(\varphi_{j}^{\nabla}(u)\right)-h_{j}\left(\psi_{j}^{\nabla}(u)\right)\right] \nabla u \\
& +\sum_{j=1}^{n} \sum_{l=1}^{n} T_{i j l}(s)\left[k_{j}\left(\varphi_{j}\left(s-\xi_{i j l}(s)\right)\right) k_{l}\left(\varphi_{l}\left(s-\zeta_{i j l}(s)\right)\right)\right. \\
& \left.-k_{j}\left(\psi_{j}\left(s-\xi_{i j l}(s)\right)\right) k_{l}\left(\psi_{l}\left(s-\zeta_{i j l}(s)\right)\right)\right], i=1,2, \ldots, n .
\end{aligned}
$$

Then, for $i=1,2, \ldots, n$, we have

$$
\begin{aligned}
\sup _{t \in \mathbb{T}}\left|x_{\varphi_{i}}(t)-x_{\psi_{i}}(t)\right|= & \sup _{t \in \mathbb{T}}\left|\int_{-\infty}^{t} \hat{e}_{-c_{i}}(t, \rho(s)) H_{i}(s, \varphi, \psi) \nabla s\right| \\
\leq & \sup _{t \in \mathbb{T}} \int_{-\infty}^{t} \hat{e}_{-c_{i}^{-}}(t, \rho(s))\left(c_{i}^{+} \delta_{i}^{+}+\sum_{j=1}^{n} a_{i j}^{+} L_{j}^{f}+\sum_{j=1}^{n} b_{i j}^{+} L_{j}^{g}\right. \\
& \left.+\sum_{j=1}^{n} d_{i j}^{+} \sigma_{i j}^{+} L_{j}^{h}+\sum_{j=1}^{n} \sum_{l=1}^{n} T_{i j l}^{+} L_{j}^{k} L_{l}^{k}\right) \nabla s\|\varphi-\psi\|_{\mathbb{B}} \\
\leq & \frac{1}{c_{i}^{-}}\left(c_{i}^{+} \delta_{i}^{+}+\sum_{j=1}^{n} a_{i j}^{+} L_{j}^{f}+\sum_{j=1}^{n} b_{i j}^{+} L_{j}^{g}+\sum_{j=1}^{n} d_{i j}^{+} \sigma_{i j}^{+} L_{j}^{h}\right.
\end{aligned}
$$




$$
\begin{aligned}
& \left.+\sum_{j=1}^{n} \sum_{l=1}^{n} T_{i j l}^{+} L_{j}^{k} L_{l}^{k}\right)\|\varphi-\psi\|_{\mathbb{B}} \\
& \sup _{t \in \mathbb{T}}\left|\left(x_{\varphi_{i}}(t)-x_{\psi_{i}}(t)\right)^{\nabla}\right|=\sup _{t \in \mathbb{T}}\left|\left(\int_{-\infty}^{t} \hat{e}_{-c_{i}}(t, \rho(s)) H_{i}(s, \varphi, \psi) \nabla s\right)^{\nabla}\right| \\
& =\sup _{t \in \mathbb{T}}\left|H_{i}(t, \varphi, \psi)-c_{i}(t) \int_{-\infty}^{t} \hat{e}_{-c_{i}}(t, \rho(s)) H_{i}(s, \varphi, \psi) \nabla s\right| \\
& \leq\left|H_{i}(t, \varphi, \psi)\right|+c_{i}^{+} \sup _{t \in \mathbb{T}}\left|\int_{-\infty}^{t} \hat{e}_{-c_{i}^{-}}(t, \rho(s)) H_{i}(s, \varphi, \psi) \nabla s\right| \\
& \leq\left(c_{i}^{+} \delta_{i}^{+}+\sum_{j=1}^{n} a_{i j}^{+} L_{j}^{f}+\sum_{j=1}^{n} b_{i j}^{+} L_{j}^{g}+\sum_{j=1}^{n} d_{i j}^{+} \sigma_{i j}^{+} L_{j}^{h}\right. \\
& \left.+\sum_{j=1}^{n} \sum_{l=1}^{n} T_{i j l}^{+} L_{j}^{k} L_{l}^{k}\right)\|\varphi-\psi\|_{\mathbb{B}} \\
& +\frac{c_{i}^{+}}{c_{i}^{-}}\left(c_{i}^{+} \delta_{i}^{+}+\sum_{j=1}^{n} a_{i j}^{+} L_{j}^{f}+\sum_{j=1}^{n} b_{i j}^{+} L_{j}^{g}+\sum_{j=1}^{n} d_{i j}^{+} \sigma_{i j}^{+} L_{j}^{h}\right. \\
& \left.+\sum_{j=1}^{n} \sum_{l=1}^{n} T_{i j l}^{+} L_{j}^{k} L_{l}^{k}\right)\|\varphi-\psi\|_{\mathbb{B}} \\
& =\frac{c_{i}^{+}+c_{i}^{-}}{c_{i}^{-}}\left(c_{i}^{+} \delta_{i}^{+}+\sum_{j=1}^{n} a_{i j}^{+} L_{j}^{f}+\sum_{j=1}^{n} b_{i j}^{+} L_{j}^{g}+\sum_{j=1}^{n} d_{i j}^{+} \sigma_{i j}^{+} L_{j}^{h}\right. \\
& \left.+\sum_{j=1}^{n} \sum_{l=1}^{n} T_{i j l}^{+} L_{j}^{k} L_{l}^{k}\right)\|\varphi-\psi\|_{\mathbb{B}}
\end{aligned}
$$

By $\left(H_{4}\right)$, we have

$$
\|\Phi(\varphi)-\Phi(\psi)\|_{\mathbb{B}}<\|\varphi-\psi\|_{\mathbb{B}} .
$$

Hence, we obtain that $\Phi$ is a contraction mapping. By the fixed point theorem of Banach space [47, it follows that $\Phi$ has a fixed point in $\mathbb{E}$; that is, system (1.1) has a unique pseudo almost periodic solution. This completes the proof of Theorem 3.1 .

\section{Global exponential stability of pseudo almost peri- odic solution}

In this section, we will study the exponential stability of pseudo almost periodic solutions of (1.1).

Definition 4.1. The pseudo almost periodic solution $x^{*}(t)=\left(x_{1}^{*}(t), x_{2}^{*}(t), \ldots, x_{n}^{*}(t)\right)^{T}$ of system (1.1) with initial value $\varphi^{*}(t)=\left(\varphi_{1}^{*}(t), \varphi_{2}^{*}(t), \ldots, \varphi_{n}^{*}(t)\right)^{T}$ is said to be globally exponentially stable if there exist a positive constant $\lambda$ with $\ominus_{\nu} \lambda \in \mathcal{R}^{+}$and $M>1$ such 
that every solution $x(t)=\left(x_{1}(t), x_{2}(t), \ldots, x_{n}(t)\right)^{T}$ of system (1.1) with initial value $\varphi(t)=$ $\left(\varphi_{1}(t), \varphi_{2}(t), \ldots, \varphi_{n}(t)\right)^{T}$ satisfies

$$
\left\|x(t)-x^{*}(t)\right\| \leq M e_{\ominus_{\nu} \lambda}\left(t, t_{0}\right)\|\psi\|, \quad \forall t \in(0,+\infty)_{\mathbb{T}}
$$

where $\|\psi\|=\sup _{t \in[-\theta, 0]_{\mathbb{T}}} \max _{1 \leq i \leq n}\left|\varphi_{i}(t)-\varphi_{i}^{*}(t)\right|, t_{0}=\max \left\{[-\theta, 0]_{\mathbb{T}}\right\}$.

Theorem 4.1. Assume that $\left(H_{1}\right)-\left(H_{4}\right)$ hold, then system (1.1) has a unique almost periodic solution that is globally exponentially stable.

Proof. From Theorem 3.1, we see that system (1.1) has at least one pseudo almost periodic solution $x^{*}(t)=\left(x_{1}^{*}(t), x_{2}^{*}(t), \ldots, x_{n}^{*}(t)\right)^{T}$ with initial value $\varphi^{*}(t)=\left(\varphi_{1}^{*}(t), \varphi_{2}^{*}(t), \ldots, \varphi_{n}^{*}(t)\right)^{T}$. Suppose that $x(t)=\left(x_{1}(t), x_{2}(t), \ldots, x_{n}(t)\right)^{T}$ is an arbitrary solution of (1.1) with initial value $\varphi(t)=\left(\varphi_{1}(t), \varphi_{2}(t), \ldots, \varphi_{n}(t)\right)^{T}$. Then it follows from system (1.1) that

$$
\begin{aligned}
z_{i}^{\nabla}(t)= & -c_{i}(t) z_{i}\left(t-\delta_{i}(t)\right)+\sum_{j=1}^{n} a_{i j}(t)\left[f_{j}\left(x_{j}(t)\right)-f_{j}\left(x_{j}^{*}(t)\right)\right]+\sum_{j=1}^{n} b_{i j}(t)\left[g_{j}\left(x_{j}\left(t-\tau_{i j}(t)\right)\right)\right. \\
& \left.-g_{j}\left(x_{j}^{*}\left(t-\tau_{i j}(t)\right)\right)\right]+\sum_{j=1}^{n} d_{i j}(t) \int_{t-\sigma_{i j}(t)}^{t}\left[h_{j}\left(x_{j}^{\nabla}(s)\right)-h_{j}\left(\left(x_{j}^{*}\right)^{\nabla}(s)\right)\right] \nabla s \\
& +\sum_{j=1}^{n} \sum_{l=1}^{n} T_{i j l}(t)\left[k_{j}\left(x_{j}\left(t-\xi_{i j l}(t)\right)\right) k_{l}\left(x_{l}\left(t-\zeta_{i j l}(t)\right)\right)\right. \\
& \left.-k_{j}\left(x_{j}^{*}\left(t-\xi_{i j l}(t)\right)\right) k_{l}\left(x_{l}^{*}\left(t-\zeta_{i j l}(t)\right)\right)\right],
\end{aligned}
$$

where $u_{i}(t)=x_{i}(t)-x_{i}^{*}(t)$ and $i=1,2, \ldots, n$.

The initial condition of (4.1) is

$$
\psi_{i}(s)=\varphi_{i}(s)-\varphi_{i}^{*}(s), \quad \psi_{j}^{\nabla}(s)=\varphi_{i}^{\Delta}(s)-\left(\varphi_{i}^{*}\right)^{\nabla}(s),
$$

where $s \in[-\theta, 0]_{\mathbb{T}}, i=1,2, \ldots, n$.

Rewrite (4.1) in the form

$$
\begin{aligned}
z_{i}^{\nabla}(t)+c_{i}(t) z_{i}(t)= & c_{i}(t) \int_{t-\delta_{i}(t)}^{t} z_{i}^{\nabla}(s) \nabla s+\sum_{j=1}^{n} a_{i j}(t)\left[f_{j}\left(x_{j}(t)\right)-f_{j}\left(x_{j}^{*}(t)\right)\right] \\
& +\sum_{j=1}^{n} b_{i j}(t)\left[g_{j}\left(x_{j}\left(t-\tau_{i j}(t)\right)\right)-g_{j}\left(x_{j}^{*}\left(t-\tau_{i j}(t)\right)\right)\right] \\
& +\sum_{j=1}^{n} d_{i j}(t) \int_{t-\sigma_{i j}(t)}^{t}\left[h_{j}\left(x_{j}^{\nabla}(s)\right)-h_{j}\left(\left(x_{j}^{*}\right)^{\nabla}(s)\right)\right] \nabla s \\
& +\sum_{j=1}^{n} \sum_{l=1}^{n} T_{i j l}(t)\left[k_{j}\left(x_{j}\left(t-\xi_{i j l}(t)\right)\right) k_{l}\left(x_{l}\left(t-\zeta_{i j l}(t)\right)\right)\right. \\
& \left.-k_{j}\left(x_{j}^{*}\left(t-\xi_{i j l}(t)\right)\right) k_{l}\left(x_{l}^{*}\left(t-\zeta_{i j l}(t)\right)\right)\right], i=1,2, \ldots, n .
\end{aligned}
$$


Multiplying the both sides of (4.2) by $\hat{e}_{-c_{i}}(t, \rho(s))$ and integrating over $\left[t_{0}, t\right]_{\mathbb{T}}$, where $t_{0} \in$ $[-\theta, 0]_{\mathbb{T}}$, by Lemma 2.4 , we get

$$
\begin{aligned}
z_{i}(t)= & z_{i}\left(t_{0}\right) \hat{e}_{-c_{i}}\left(t, t_{0}\right)+\int_{t_{0}}^{t} \hat{e}_{-c_{i}}(t, \rho(s))\left(c_{i}(s) \int_{s-\delta_{i}(s)}^{s} z_{i}^{\nabla}(u) \nabla u\right. \\
& +\sum_{j=1}^{n} a_{i j}(s)\left[f_{j}\left(x_{j}(s)\right)-f_{j}\left(x_{j}^{*}(s)\right)\right]+\sum_{j=1}^{n} b_{i j}(s)\left[g_{j}\left(x_{j}\left(s-\tau_{i j}(s)\right)\right)\right. \\
& \left.-g_{j}\left(x_{j}^{*}\left(s-\tau_{i j}(s)\right)\right)\right]+\sum_{j=1}^{n} d_{i j}(s) \int_{s-\sigma_{i j}(s)}^{s}\left[h_{j}\left(x_{j}^{\nabla}(u)\right)-h_{j}\left(\left(x_{j}^{*}\right)^{\nabla}(u)\right)\right] \nabla u \\
& +\sum_{j=1}^{n} \sum_{l=1}^{n} T_{i j l}(s)\left[k_{j}\left(x_{j}\left(s-\xi_{i j l}(s)\right)\right) k_{l}\left(x_{l}\left(s-\zeta_{i j l}(s)\right)\right)\right. \\
& \left.\left.-k_{j}\left(x_{j}^{*}\left(s-\xi_{i j l}(s)\right)\right) k_{l}\left(x_{l}^{*}\left(s-\zeta_{i j l}(s)\right)\right)\right]\right) \nabla s, i=1,2, \ldots, n .
\end{aligned}
$$

Let $R_{i}$ be defined as follows:

$$
\begin{aligned}
R_{i}(\beta)= & c_{i}^{-}-\beta-\left(c_{i}^{+} \exp \left(\beta \sup _{s \in \mathbb{T}} \nu(s)\right)+c_{i}^{-}-\beta\right)\left(c_{i}^{+} \delta_{i}^{+} \exp \left(\beta \delta_{i}^{+}\right)\right. \\
& +\sum_{j=1}^{n} a_{i j}^{+} L_{j}^{f}+\sum_{j=1}^{n} b_{i j}^{+} L_{j}^{g} \exp \left(\beta \tau_{i j}^{+}\right)+\sum_{j=1}^{n} d_{i j}^{+} L_{j}^{h} \sigma_{i j}^{+} \exp \left(\beta \sigma_{i j}^{+}\right) \\
& \left.+\sum_{j=1}^{n} \sum_{l=1}^{n} T_{i j l}^{+} L_{j}^{k} L_{l}^{k}\left(\exp \left(\beta \xi_{i j l}^{+}\right)+\exp \left(\beta \zeta_{i j l}^{+}\right)\right)\right), i=1,2, \ldots, n .
\end{aligned}
$$

By $\left(H_{4}\right)$, we get

$$
\begin{aligned}
R_{i}(0)= & c_{i}^{-}-\left(c_{i}^{+} \delta_{i}^{+}+\sum_{j=1}^{n} a_{i j}^{+} L_{j}^{f}+\sum_{j=1}^{n} b_{i j}^{+} L_{j}^{g}+\sum_{j=1}^{n} d_{i j}^{+} L_{j}^{h}\right. \\
& \left.+\sum_{j=1}^{n} \sum_{l=1}^{n} T_{i j l}^{+} L_{j}^{k} L_{l}^{k}\right)>0, i=1,2, \ldots, n .
\end{aligned}
$$

Since $R_{i}$ is continuous on $[0,+\infty)$ and $R_{i}(\beta) \rightarrow-\infty$, as $\beta \rightarrow+\infty$, there exists $\gamma_{i}>0$ such that $R_{i}\left(\gamma_{i}\right)=0$ and $R_{i}(\beta)>0$ for $\beta \in\left(0, \gamma_{i}\right)$. Take $a=\min _{1 \leq i \leq n}\left\{\gamma_{i}\right\}$, we have $R_{i}(a) \geq 0$. So, we can choose a positive constant $0<\lambda<\min \left\{a, \min _{1 \leq i \leq n}\left\{c_{i}^{-}\right\}\right\}$such that

$$
R_{i}(\lambda)>0, \quad i=1,2, \ldots, n
$$

which implies that

$$
\left(1+\frac{c_{i}^{+} \exp \left(\lambda \sup _{s \in \mathbb{T}} \nu(s)\right)}{c_{i}^{-}-\lambda}\right)\left(c_{i}^{-}-\left(c_{i}^{+} \delta_{i}^{+}+\sum_{j=1}^{n} a_{i j}^{+} L_{j}^{f}+\sum_{j=1}^{n} b_{i j}^{+} L_{j}^{g}+\sum_{j=1}^{n} d_{i j}^{+} L_{j}^{h}\right.\right.
$$




$$
\left.\left.+\sum_{j=1}^{n} \sum_{l=1}^{n} T_{i j l}^{+} L_{j}^{k} L_{l}^{k}\right)\right)<1, i=1,2, \ldots, n
$$

Let

$$
M=\max _{1 \leq i \leq n}\left\{\frac{c_{i}^{-}}{c_{i}^{-}-\left(c_{i}^{+} \delta_{i}^{+}+\sum_{j=1}^{n} a_{i j}^{+} L_{j}^{f}+\sum_{j=1}^{n} b_{i j}^{+} L_{j}^{g}+\sum_{j=1}^{n} d_{i j}^{+} L_{j}^{h}+\sum_{j=1}^{n} \sum_{l=1}^{n} T_{i j l}^{+} L_{j}^{k} L_{l}^{k}\right)}\right\},
$$

then by $\left(H_{4}\right)$ we have $M>1$.

Hence, it is obvious that

$$
\|z(t)\|_{\mathbb{B}} \leq M \hat{e}_{\ominus_{\nu} \lambda}\left(t, t_{0}\right)\|\psi\|_{\mathbb{B}}, \quad \forall t \in\left[-\theta, t_{0}\right]_{\mathbb{T}},
$$

where $\ominus_{\nu} \lambda \in \mathcal{R}^{+}$. We claim that

$$
\|z(t)\|_{\mathbb{B}} \leq M \hat{e}_{\ominus_{\nu} \lambda}\left(t, t_{0}\right)\|\psi\|_{\mathbb{B}}, \quad \forall t \in\left(t_{0},+\infty\right)_{\mathbb{T}} .
$$

To prove (4.6), we show that for any $P>1$, the following inequality holds:

$$
\|z(t)\|_{\mathbb{B}}<P M \hat{e}_{\ominus \nu \lambda}\left(t, t_{0}\right)\|\psi\|_{\mathbb{B}}, \quad \forall t \in\left(t_{0},+\infty\right)_{\mathbb{T}}
$$

which implies that, for $i=1,2, \ldots, n$, we have

$$
\left|z_{i}(t)\right|<P M \hat{e}_{\ominus \nu \lambda}\left(t, t_{0}\right)\|\psi\|_{\mathbb{B}}, \quad \forall t \in\left(t_{0},+\infty\right)_{\mathbb{T}}
$$

and

$$
\left|z_{i}^{\nabla}(t)\right|<P M \hat{e}_{\ominus_{\nu} \lambda}\left(t, t_{0}\right)\|\psi\|_{\mathbb{B}}, \quad \forall t \in\left(t_{0},+\infty\right)_{\mathbb{T}} .
$$

If (4.7) is not true, then there must be some $t_{1} \in\left(t_{0},+\infty\right)_{\mathbb{T}}$ and some $i_{1}, i_{2} \in\{1,2, \ldots, n\}$ such that

$$
\left\|z\left(t_{1}\right)\right\|_{\mathbb{B}}=\max \left\{\left|z_{i_{1}}\left(t_{1}\right)\right|,\left|z_{i_{2}}^{\nabla}\left(t_{1}\right)\right|\right\} \geq P M \hat{e}_{\ominus_{\nu} \lambda}\left(t_{1}, t_{0}\right)\|\psi\|_{\mathbb{B}}
$$

and

$$
\|z(t)\|_{\mathbb{B}} \leq P M \hat{e}_{\ominus_{\nu} \lambda}\left(t, t_{0}\right)\|\psi\|_{\mathbb{B}}, \quad t \in\left(t_{0}, t_{1}\right]_{\mathbb{T}}, t_{0} \in[-\theta, 0]_{\mathbb{T}}
$$

Therefore, there must exist a constant $c \geq 1$ such that

$$
\left\|z\left(t_{1}\right)\right\|_{\mathbb{B}}=\max \left\{\left|z_{i_{1}}\left(t_{1}\right)\right|,\left|z_{i_{2}}^{\nabla}\left(t_{1}\right)\right|\right\}=c P M \hat{e}_{\ominus_{\nu} \lambda}\left(t_{1}, t_{0}\right)\|\psi\|_{\mathbb{B}}
$$

and

$$
\|z(t)\|_{\mathbb{B}} \leq c P M \hat{e}_{\ominus_{\nu} \lambda}\left(t, t_{0}\right)\|\psi\|_{\mathbb{B}}, \quad t \in\left(t_{0}, t_{1}\right]_{\mathbb{T}}, t_{0} \in[-\theta, 0]_{\mathbb{T}} .
$$


In view of (4.3), we have

$$
\begin{aligned}
& \left|z_{i_{1}}\left(t_{1}\right)\right| \\
& =\mid z_{i_{1}}\left(t_{0}\right) e_{-c_{i_{1}}}\left(t_{1}, t_{0}\right)+\int_{t_{0}}^{t_{1}} e_{-c_{i_{1}}}\left(t_{1}, \rho(s)\right)\left(c_{i_{1}}(s) \int_{s-\delta_{i_{1}}(s)}^{s} z_{i_{1}}^{\nabla}(u) \nabla u\right. \\
& +\sum_{j=1}^{n} a_{i_{1} j}(s)\left[f_{j}\left(x_{j}(s)\right)-f_{j}\left(x_{j}^{*}(s)\right)\right]+\sum_{j=1}^{n} b_{i_{1} j}(s)\left[g_{j}\left(x_{j}\left(s-\tau_{i_{1} j}(s)\right)\right)\right. \\
& \left.-g_{j}\left(x_{j}^{*}\left(s-\tau_{i_{1} j}(s)\right)\right)\right]+\sum_{j=1}^{n} d_{i_{1} j}(s) \int_{s-\sigma_{i_{1} j}(s)}^{s}\left[h_{j}\left(x_{j}^{\nabla}(u)\right)-h_{j}\left(\left(x_{j}^{*}\right)^{\nabla}(u)\right)\right] \nabla u \\
& +\sum_{j=1}^{n} \sum_{l=1}^{n} T_{i_{1} j l}(s)\left[k_{j}\left(x_{j}\left(s-\xi_{i_{1} j l}(s)\right)\right) k_{l}\left(x_{l}\left(s-\zeta_{i_{1} j l}(s)\right)\right)\right. \\
& \left.\left.-k_{j}\left(x_{j}^{*}\left(s-\xi_{i_{1 j l}}(s)\right)\right) k_{l}\left(x_{l}^{*}\left(s-\zeta_{i_{1} j l}(s)\right)\right)\right]\right) \nabla s \mid \\
& \leq \hat{e}_{-c_{i_{1}}}\left(t_{1}, t_{0}\right)\|\psi\|_{\mathbb{B}}+c P M \hat{e}_{\ominus_{\nu} \lambda}\left(t_{1}, t_{0}\right)\|\psi\|_{\mathbb{B}} \int_{t_{0}}^{t_{1}} \hat{e}_{-c_{i_{1}}}\left(t_{1}, \rho(s)\right) \hat{e}_{\lambda}\left(t_{1}, \rho(s)\right) \\
& \times\left(c_{i_{1}}^{+} \int_{s-\delta_{i_{1}}(s)}^{s} \hat{e}_{\lambda}(\rho(u), u) \nabla u+\sum_{j=1}^{n} a_{i_{1} j}^{+} L_{j}^{f} \hat{e}_{\lambda}(\rho(s), s)+\sum_{j=1}^{n} b_{i_{1} j}^{+} L_{j}^{g} \hat{e}_{\lambda}\left(\rho(s), s-\tau_{i_{1} j}(s)\right)\right. \\
& +\sum_{j=1}^{n} d_{i_{1} j}^{+} L_{j}^{h} \int_{s-\sigma_{i_{1} j}(s)}^{s} \hat{e}_{\lambda}(\rho(u), u) \nabla u+\sum_{j=1}^{n} \sum_{l=1}^{n} T_{i_{1} j l}^{+} L_{j}^{k} L_{l}^{k}\left(\hat{e}_{\lambda}\left(\rho(s), s-\xi_{i_{1} j l}(s)\right)\right. \\
& \left.\left.+\hat{e}_{\lambda}\left(\rho(s), s-\zeta_{i_{1 j} l}(s)\right)\right)\right) \nabla s \\
& \leq \hat{e}_{-c_{i_{1}}}\left(t_{1}, t_{0}\right)\|\psi\|_{\mathbb{B}}+c P M \hat{e}_{\ominus_{\nu} \lambda}\left(t_{1}, t_{0}\right)\|\psi\|_{\mathbb{B}} \int_{t_{0}}^{t_{1}} \hat{e}_{-c_{i_{1}} \oplus_{\nu} \lambda}\left(t_{1}, \rho(s)\right) \\
& \times\left(c_{i_{1}}^{+} \delta_{i_{1}}^{+} \hat{e}_{\lambda}\left(\rho(s), s-\delta_{i_{1}}(s)\right)+\sum_{j=1}^{n} a_{i_{1 j}}^{+} L_{j}^{f} \hat{e}_{\lambda}(\rho(s), s)+\sum_{j=1}^{n} b_{i_{1} j}^{+} L_{j}^{g} \hat{e}_{\lambda}\left(\rho(s), s-\tau_{i_{1} j}(s)\right)\right. \\
& +\sum_{j=1}^{n} d_{i_{1} j}^{+} L_{j}^{h} \sigma_{i_{1} j}^{+} \hat{e}_{\lambda}\left(\rho(s), s-\sigma_{i_{1} j}(s)\right)+\sum_{j=1}^{n} \sum_{l=1}^{n} T_{i_{1} j l}^{+} L_{j}^{k} L_{l}^{k}\left(\hat{e}_{\lambda}\left(\rho(s), s-\xi_{i_{1} j l}(s)\right)\right. \\
& \left.\left.+\hat{e}_{\lambda}\left(\rho(s), s-\zeta_{i_{1} j l}(s)\right)\right)\right) \nabla s \\
& \leq \hat{e}_{-c_{i_{1}}}\left(t_{1}, t_{0}\right)\|\psi\|_{\mathbb{B}}+c P M \hat{e}_{\ominus_{\nu} \lambda}\left(t_{1}, t_{0}\right)\|\psi\|_{\mathbb{B}} \int_{t_{0}}^{t_{1}} \hat{e}_{-c_{i_{1}} \oplus_{\nu} \lambda}\left(t_{1}, \rho(s)\right) \\
& \times\left(c_{i_{1}}^{+} \delta_{i_{1}}^{+} \exp \left[\lambda\left(\delta_{i_{1}}^{+}+\sup _{s \in \mathbb{T}} \nu(s)\right)\right]+\sum_{j=1}^{n} a_{i_{1} j}^{+} L_{j}^{f} \exp \left(\lambda \sup _{s \in \mathbb{T}} \nu(s)\right)\right.
\end{aligned}
$$




$$
\begin{aligned}
& +\sum_{j=1}^{n} b_{i_{1} j}^{+} L_{j}^{g} \exp \left[\lambda\left(\tau_{i_{1} j}^{+}+\sup _{s \in \mathbb{T}} \nu(s)\right)\right]+\sum_{j=1}^{n} d_{i_{1} j}^{+} L_{j}^{h} \sigma_{i_{1} j}^{+} \exp \left[\lambda\left(\sigma_{i_{1} j}^{+}+\sup _{s \in \mathbb{T}} \nu(s)\right)\right] \\
& \left.+\sum_{j=1}^{n} \sum_{l=1}^{n} T_{i_{1} j l}^{+} L_{j}^{k} L_{l}^{k}\left(\exp \left[\lambda\left(\xi_{i_{1} j}^{+}+\sup _{s \in \mathbb{T}} \nu(s)\right)\right]+\exp \left[\lambda\left(\zeta_{i_{1} j}^{+}+\sup _{s \in \mathbb{T}} \nu(s)\right)\right]\right)\right) \nabla s \\
& =c P M \hat{e}_{\ominus_{\nu} \lambda}\left(t_{1}, t_{0}\right)\|\psi\|_{\mathbb{B}}\left\{\frac{1}{p M} \hat{e}_{-c_{i_{1}} \oplus_{\nu} \lambda}\left(t_{1}, t_{0}\right)+\exp \left(\lambda \sup _{s \in \mathbb{T}} \nu(s)\right)\right. \\
& \times\left(c_{i_{1}}^{+} \delta_{i_{1}}^{+} \exp \left(\lambda \delta_{i_{1}}^{+}\right)+\sum_{j=1}^{n} a_{i_{1} j}^{+} L_{j}^{f}+\sum_{j=1}^{n} b_{i_{1} j}^{+} L_{j}^{g} \exp \left(\lambda \tau_{i_{1} j}^{+}\right)\right. \\
& \left.+\sum_{j=1}^{n} d_{i_{1} j}^{+} L_{j}^{h} \sigma_{i_{1} j}^{+} \exp \left(\lambda \sigma_{i_{1} j}^{+}\right)+\sum_{j=1}^{n} \sum_{l=1}^{n} T_{i_{1} j l}^{+} L_{j}^{k} L_{l}^{k}\left(\exp \left(\lambda \xi_{i_{1} j}^{+}\right)+\exp \left(\lambda \zeta_{i_{1} j}^{+}\right)\right)\right) \\
& \left.\times \int_{t_{0}}^{t_{1}} \hat{e}_{-c_{i_{1}} \oplus_{\nu} \lambda}\left(t_{1}, \rho(s)\right)\right\} \nabla s \\
& \leq c P M \hat{e}_{\ominus_{\nu} \lambda}\left(t_{1}, t_{0}\right)\|\psi\|_{\mathbb{B}}\left\{\frac{1}{p M} \hat{e}_{-c_{i_{1}} \oplus_{\nu} \lambda}\left(t_{1}, t_{0}\right)+\exp \left(\lambda \sup _{s \in \mathbb{T}} \nu(s)\right)\right. \\
& \times\left(c_{i_{1}}^{+} \delta_{i_{1}}^{+} \exp \left(\lambda \delta_{i_{1}}^{+}\right)+\sum_{j=1}^{n} a_{i_{1} j}^{+} L_{j}^{f}+\sum_{j=1}^{n} b_{i_{1} j}^{+} L_{j}^{g} \exp \left(\lambda \tau_{i_{1} j}^{+}\right)\right. \\
& \left.+\sum_{j=1}^{n} d_{i_{1} j}^{+} L_{j}^{h} \sigma_{i_{1} j}^{+} \exp \left(\lambda \sigma_{i_{1} j}^{+}\right)+\sum_{j=1}^{n} \sum_{l=1}^{n} T_{i_{1} j l}^{+} L_{j}^{k} L_{l}^{k}\left(\exp \left(\lambda \xi_{i_{1} j}^{+}\right)+\exp \left(\lambda \zeta_{i_{1} j}^{+}\right)\right)\right) \\
& \left.\times \frac{1-\hat{e}_{-c_{i_{1}} \oplus \nu}\left(t_{1}, t_{0}\right)}{c_{i_{1}}^{-}-\lambda}\right\} \\
& \leq c P M \hat{e}_{\ominus_{\nu} \lambda}\left(t_{1}, t_{0}\right)\|\psi\|_{\mathbb{B}}\left\{\left[\frac{1}{M}-\frac{\exp \left(\lambda \sup _{s \in \mathbb{T}} \nu(s)\right)}{c_{i_{1}}^{-}-\lambda}\left(c_{i_{1}}^{+} \delta_{i_{1}}^{+} \exp \left(\lambda \delta_{i_{1}}^{+}\right)\right.\right.\right. \\
& +\sum_{j=1}^{n} a_{i_{1 j}}^{+} L_{j}^{f}+\sum_{j=1}^{n} b_{i_{1 j}}^{+} L_{j}^{g} \exp \left(\lambda \tau_{i_{1} j}^{+}\right)+\sum_{j=1}^{n} d_{i_{1} j}^{+} L_{j}^{h} \sigma_{i_{1} j}^{+} \exp \left(\lambda \sigma_{i_{1} j}^{+}\right) \\
& \left.\left.+\sum_{j=1}^{n} \sum_{l=1}^{n} T_{i_{1} j l}^{+} L_{j}^{k} L_{l}^{k}\left(\exp \left(\lambda \xi_{i_{1} j}^{+}\right)+\exp \left(\lambda \zeta_{i_{1} j}^{+}\right)\right)\right)\right] \hat{e}_{-c_{i_{1}} \oplus_{\nu} \lambda}\left(t_{1}, t_{0}\right) \\
& +\frac{\exp \left(\lambda \sup _{s \in \mathbb{T}} \nu(s)\right)}{c_{i_{1}}^{-}-\lambda}\left(c_{i_{1}}^{+} \delta_{i_{1}}^{+} \exp \left(\lambda \delta_{i_{1}}^{+}\right)+\sum_{j=1}^{n} a_{i_{1} j}^{+} L_{j}^{f}+\sum_{j=1}^{n} b_{i_{1 j}}^{+} L_{j}^{g} \exp \left(\lambda \tau_{i_{1 j}}^{+}\right)\right. \\
& \left.\left.+\sum_{j=1}^{n} d_{i_{1} j}^{+} L_{j}^{h} \sigma_{i_{1} j}^{+} \exp \left(\lambda \sigma_{i_{1} j}^{+}\right)+\sum_{j=1}^{n} \sum_{l=1}^{n} T_{i_{1} j l}^{+} L_{j}^{k} L_{l}^{k}\left(\exp \left(\lambda \xi_{i_{1} j}^{+}\right)+\exp \left(\lambda \zeta_{i_{1} j}^{+}\right)\right)\right)\right\} \\
& \leq c P M \hat{e}_{\ominus_{\nu} \lambda}\left(t_{1}, t_{0}\right)\|\psi\|_{\mathbb{B}}
\end{aligned}
$$


and

$$
\begin{aligned}
& \left|z_{i_{2}}^{\nabla}\left(t_{1}\right)\right| \leq c_{i_{2}}^{+} \hat{e}_{-c_{i_{2}}}\left(t_{1}, t_{0}\right)\|\psi\|_{\mathbb{B}}+c P M \hat{e}_{\ominus_{\nu} \lambda}\left(t_{1}, t_{0}\right)\|\psi\|_{\mathbb{B}}\left(c_{i_{2}}^{+} \int_{t_{1}-\delta_{i_{2}}\left(t_{1}\right)}^{t_{1}} e_{\lambda}\left(t_{1}, u\right) \nabla u\right. \\
& +\sum_{j=1}^{n} a_{i_{2} j}^{+} L_{j}^{f} \hat{e}_{\lambda}\left(t_{1}, t_{1}\right)+\sum_{j=1}^{n} b_{i_{2} j}^{+} L_{j}^{g} \hat{e}_{\lambda}\left(t_{1}, t_{1}-\tau_{i_{2} j}\left(t_{1}\right)\right) \\
& +\sum_{j=1}^{n} d_{i_{2} j}^{+} L_{j}^{h} \int_{s-\sigma_{i_{2} j}(s)}^{s} \hat{e}_{\lambda}(\rho(u), u) \nabla u \\
& \left.+\sum_{j=1}^{n} \sum_{l=1}^{n} T_{i_{2 j l}}^{+} L_{j}^{k} L_{l}^{k}\left(\hat{e}_{\lambda}\left(t_{1}, t_{1}-\xi_{i_{2} j l}\left(t_{1}\right)\right)+\hat{e}_{\lambda}\left(t_{1}, t_{1}-\zeta_{i_{2} j l}\left(t_{1}\right)\right)\right)\right) \\
& +c_{i_{2}}^{+} c P M \hat{e}_{\ominus_{\nu} \lambda}\left(t_{1}, t_{0}\right)\|\psi\|_{\mathbb{B}} \int_{t_{0}}^{t_{1}} \hat{e}_{-c_{i_{2}}}\left(t_{1}, \rho(s)\right) \hat{e}_{\lambda}\left(t_{1}, \rho(s)\right) \\
& \times\left\{c_{i_{2}}^{+} \int_{s-\eta_{i_{2}}(s)}^{s} \hat{e}_{\lambda}(\rho(s), u) \nabla u+\sum_{j=1}^{n} a_{i_{2} j}^{+} L_{j}^{f} \hat{e}_{\lambda}(\rho(s), s)\right. \\
& +\sum_{j=1}^{n} b_{i_{2} j}^{+} L_{j}^{g} \hat{e}_{\lambda}\left(\rho(s), s-\tau_{i_{2} j}(s)\right)+\sum_{j=1}^{n} d_{i_{2} j}^{+} L_{j}^{h} \int_{s-\sigma_{i_{2} j}(s)}^{s} \hat{e}_{\lambda}(\rho(u), u) \nabla u \\
& \left.+\sum_{j=1}^{n} \sum_{l=1}^{n} T_{i_{2} j l}^{+} L_{j}^{k} L_{l}^{k}\left(\hat{e}_{\lambda}\left(s, t_{1}-\xi_{i_{2} j l}(s)\right)+\hat{e}_{\lambda}\left(s, t_{1}-\zeta_{i_{2} j l}(s)\right)\right)\right\} \nabla s \\
& \leq c_{i_{2}}^{+} e_{-c_{i_{2}}}\left(t_{1}, t_{0}\right)\|\psi\|_{\mathbb{B}}+c P M \hat{e}_{\ominus_{\nu} \lambda}\left(t_{1}, t_{0}\right)\|\psi\|_{\mathbb{B}}\left(c_{i_{2}}^{+} \delta_{i_{2}}^{+} \exp \left(\lambda \delta_{i_{2}}^{+}\right)+\sum_{j=1}^{n} a_{i_{2} j}^{+} L_{j}^{f}\right. \\
& +\sum_{j=1}^{n} b_{i_{2} j}^{+} L_{j}^{g} \exp \left(\lambda \tau_{i_{2} j}^{+}\right)+\sum_{j=1}^{n} d_{i_{2} j}^{+} L_{j}^{h} \sigma_{i_{2} j}^{+} \exp \left(\lambda \sigma_{i_{2} j}^{+}\right) \\
& \left.+\sum_{j=1}^{n} \sum_{l=1}^{n} T_{i_{2} j l}^{+} L_{j}^{k} L_{l}^{k}\left(\exp \left(\lambda \xi_{i_{2} j l}^{+}\right)+\exp \left(\lambda \zeta_{i_{2} j l}^{+}\right)\right)\right) \\
& \times\left(1+c_{i_{2}}^{+} \exp \left(\lambda \sup _{s \in \mathbb{T}} \nu(s)\right) \int_{t_{0}}^{t_{1}} \hat{e}_{-c_{i_{2}} \oplus \lambda}\left(t_{1}, \rho(s)\right) \Delta s\right) \\
& \leq c P M \hat{e}_{\ominus_{\nu} \lambda}\left(t_{1}, t_{0}\right)\|\psi\|_{\mathbb{B}}\left\{\frac{c_{i_{2}}^{+}}{M} \hat{e}_{-\left(c_{i_{2}}^{-}-\lambda\right)}\left(t_{1}, t_{0}\right)+\left(c_{i_{2}}^{+} \delta_{i_{2}}^{+} \exp \left(\lambda \delta_{i_{2}}^{+}\right)+\sum_{j=1}^{n} a_{i_{2} j}^{+} L_{j}^{f}\right.\right. \\
& +\sum_{j=1}^{n} b_{i_{2} j}^{+} L_{j}^{g} \exp \left(\lambda \tau_{i_{2} j}^{+}\right)+\sum_{j=1}^{n} d_{i_{2} j}^{+} L_{j}^{h} \sigma_{i_{2} j}^{+} \exp \left(\lambda \sigma_{i_{2} j}^{+}\right) \\
& \left.+\sum_{j=1}^{n} \sum_{l=1}^{n} T_{i_{2} j l}^{+} L_{j}^{k} L_{l}^{k}\left(\exp \left(\lambda \xi_{i_{2} j l}^{+}\right)+\exp \left(\lambda \zeta_{i_{2} j l}^{+}\right)\right)\right)
\end{aligned}
$$




$$
\begin{aligned}
& \left.\times\left(1+c_{i_{2}}^{+} \exp \left(\lambda \sup _{s \in \mathbb{T}} \nu(s)\right) \frac{1}{-\left(c_{i_{2}}^{-}-\lambda\right)}\left(\hat{e}_{-\left(c_{i_{2}}^{-}-\lambda\right)}\left(t_{1}, t_{0}\right)-1\right)\right)\right\} \\
\leq & c P M \hat{e}_{\ominus \nu}\left(t_{1}, t_{0}\right)\|\psi\|_{\mathbb{B}}\left\{\left[\frac{1}{M}-\frac{\exp \left(\lambda \sup _{s \in \mathbb{T}} \nu(s)\right)}{c_{i_{2}}^{-}-\lambda}\left(c_{i_{2}}^{+} \delta_{i_{2}}^{+} \exp \left(\lambda \delta_{i_{2}}^{+}\right) \sum_{j=1}^{n} a_{i_{2} j}^{+} L_{j}^{f}\right.\right.\right. \\
& +\sum_{j=1}^{n} b_{i_{2} j}^{+} L_{j}^{g} \exp \left(\lambda \tau_{i_{2} j}^{+}\right)+\sum_{j=1}^{n} d_{i_{2} j}^{+} L_{j}^{h} \sigma_{i_{2} j}^{+} \exp \left(\lambda \sigma_{i_{2} j}^{+}\right) \\
& \left.\left.+\sum_{j=1}^{n} \sum_{l=1}^{n} T_{i_{2} j l}^{+} L_{j}^{k} L_{l}^{k}\left(\exp \left(\lambda \xi_{i_{2} j l}^{+}\right)+\exp \left(\lambda \zeta_{i_{2} j l}^{+}\right)\right)\right)\right] c_{i_{2}}^{+} \hat{e}_{-\left(i_{i_{2}}-\lambda\right)}\left(t_{1}, t_{0}\right) \\
& +\left(1+\frac{c_{i_{2}}^{+} \exp \left(\lambda \sup _{s \in \mathbb{T}} \mu(s)\right)}{c_{i_{2}}^{-}-\lambda}\right)\left(c_{i_{2}}^{+} \delta_{i_{2}}^{+} \exp \left(\lambda \delta_{i_{2}}^{+}\right)\right. \\
& +\sum_{j=1}^{n} a_{i_{2} j}^{+} L_{j}^{f}+\sum_{j=1}^{n} b_{i_{2} j}^{+} L_{j}^{g} \exp \left(\lambda \tau_{i_{2} j}^{+}\right)+\sum_{j=1}^{n} d_{i_{2} j}^{+} L_{j}^{h} \sigma_{i_{2} j}^{+} \exp \left(\lambda \sigma_{i_{2} j}^{+}\right) \\
& \left.\left.+\sum_{j=1}^{n} \sum_{l=1}^{n} T_{i_{2} j l}^{+} L_{j}^{k} L_{l}^{k}\left(\exp \left(\lambda \xi_{i_{2} j l}^{+}\right)+\exp \left(\lambda \zeta_{i_{2} j l}^{+}\right)\right)\right)\right\} \\
< & c P M \hat{e}_{\ominus} \lambda\left(t_{1}, t_{0}\right)\|\psi\|_{\mathbb{B} .}
\end{aligned}
$$

The above two inequalities imply that

$$
\left\|z\left(t_{1}\right)\right\|_{\mathbb{B}}<c P M \hat{e}_{\ominus_{\nu} \lambda}\left(t_{1}, t_{0}\right)\|\psi\|_{\mathbb{B}},
$$

which contradicts (4.10), and so (4.7) holds. Letting $P \rightarrow 1$, then (4.6) holds. Hence, the pseudo almost periodic solution of system (1.1) is globally exponentially stable. The proof is complete.

\section{$5 \quad$ An example}

In this section, we will give an example to illustrate the feasibility and effectiveness of our results obtained in Sections 3 and 4.

Example 5.1. Let $n=2$. Consider the following neural network system on time scale $\mathbb{T}$ :

$$
\begin{aligned}
x_{i}^{\nabla}(t)= & -c_{i}(t) x_{i}\left(t-\delta_{i}(t)\right)+\sum_{j=1}^{2} a_{i j}(t) f_{j}\left(x_{j}(t)\right) \\
& +\sum_{j=1}^{2} b_{i j}(t) g_{j}\left(x_{j}\left(t-\tau_{i j}(t)\right)\right)+\sum_{j=1}^{2} d_{i j}(t) \int_{t-\sigma_{i j}(t)}^{t} h_{j}\left(x_{j}^{\nabla}(s)\right) \nabla s \\
& +\sum_{j=1}^{2} \sum_{l=1}^{2} T_{i j l}(t) k_{j}\left(x_{j}\left(t-\xi_{i j l}(t)\right)\right) k_{l}\left(x_{l}\left(t-\zeta_{i j l}(t)\right)\right)+I_{i}(t),
\end{aligned}
$$


where $t \in \mathbb{T}, i=1,2$ and the coefficients are as follows:

$$
\begin{gathered}
c_{1}(t)=0.95+0.05 \sin t, \quad c_{2}(t)=0.91+0.04 \sin t, \quad a_{11}(t)=0.05 \cos t \\
a_{12}(t)=0.07 \cos \sqrt{2} t, \quad a_{21}(t)=0.05 \cos \left(\frac{1}{3} t\right), \quad a_{22}(t)=0.03 \cos \left(\frac{3}{4} t\right), \\
b_{11}(t)=0.06 \sin t, \quad b_{12}(t)=0.03 \cos \sqrt{2} t, \quad b_{21}(t)=0.04 \cos t, \\
b_{22}(t)=0.03 \sin \sqrt{2} t, \quad d_{11}(t)=0.08 \sin t, \quad d_{12}(t)=0.04 \sin \sqrt{2} t, \quad d_{21}(t)=0.06 \sin t, \\
d_{22}(t)=0.07 \cos \sqrt{2} t, \quad f_{1}(u)=0.1 \sin u, \quad f_{2}(u)=\sin u, \quad g_{1}(u)=0.1 \cos u, \quad g_{2}(u)=\cos u, \\
h_{1}(u)=0.1 \sin \frac{1}{3} u, \quad h_{2}(u)=\sin \sqrt{u}, \quad k_{1}(u)=0.1 \cos \sqrt{2 u}, \quad k_{2}(u)=\sin 3 u, \\
T_{111}(t)=T_{222}(t)=0.075+0.025 \sin \sqrt{2} t, T_{112}(t)=T_{212}(t)=0.07+0.03 \cos \left(\frac{3}{4} t\right), \\
T_{121}(t)=T_{221}(t)=0.075+0.025 \cos \sqrt{3} t, T_{122}(t)=T_{211}(t)=0.07+0.03 \sin \left(\frac{3}{4} t\right) . \\
I_{1}(t)=I_{2}(t)=0.5 \sin 2 t, \quad J_{1}(t)=J_{2}(t)=0.5 \cos \sqrt{2} t .
\end{gathered}
$$

By a simple calculation, we have

$$
\begin{gathered}
c_{1}^{+}=0.1, \quad c_{2}^{+}=0.95, \quad c_{1}^{-}=0.9, \quad c_{2}^{-}=0.0 .87, \\
a_{11}^{+}=0.05, \quad a_{12}^{+}=0.07, \quad a_{21}^{+}=0.05, \quad a_{22}^{+}=0.03, \\
b_{11}^{+}=0.06, \quad b_{12}^{+}=0.03, \quad b_{21}^{+}=0.04, \quad b_{22}^{+}=0.03, \\
d_{11}^{+}=0.08, \quad d_{12}^{+}=0.04, \quad d_{21}^{+}=0.06, \quad d_{22}^{+}=0.07, \\
T_{111}^{+}=T_{222}^{+}=T_{112}^{+}=T_{212}^{+}=T_{121}^{+}=T_{221}^{+}=T_{122}^{+}=T_{211}^{+}=0.01, \\
H_{1}^{f}=H_{1}^{g}=H_{1}^{h}=H_{1}^{k}=0.1, \quad H_{2}^{f}=H_{2}^{g}=H_{2}^{h}=H_{2}^{k}=1 .
\end{gathered}
$$

Therefore, whether $\mathbb{T}=\mathbb{R}$ or $\mathbb{T}=\mathbb{Z}$, all the conditions of Theorem 3.1 and Theorem 4.1 are satisfied, hence, we know that system (5.1) has a pseudo almost periodic solution, which is globally exponentially stable. This is, the continuous-time neural network and its discrete-time analogue have the same dynamical behaviors for the pseudo almost periodicity. 


\section{References}

[1] Z.H. Guan, D.B. Sun, J.J. Shen, Qualitative analysis of high-order Hopfield neural networks, Acta Electron. Sin. 28 (2000) 77-80.

[2] B.J. Xu, X.Z. Liu, X.X. Liao, Global asymptonic stability of high-order Hopfield neural networks with time delays, Comput. Math. Appl. 45 (2003) 1729-1737.

[3] B.J. Xu, X.Z. Liu, X.X. Liao, Global asymptotic stability of high-order Hopfield type neural networks with time delays, Comput. Math. Appl. 174 (2006) 98-116.

[4] X.Y. Lou, B.T. Cui, Novel global stability criteria for high-order Hopfield-type neural networks with time-varying delays, J. Math. Anal. Appl. 330 (2007) 144-158.

[5] F. Zhang, Y. Li, Almost periodic solutions for higher-order Hopfield neural networks without bounded activation functions, Electron. J. Diff. Eqns. 2007 (97) (2007) 1-10.

[6] Y.H. Yua, M.S. Cai, Existence and exponential stability of almost-periodic solutions for high-order Hopfield neural networks, Math. Comput. Modelling 47 (2008) 943-951.

[7] C.X. Ou, Anti-periodic solutions for high-order Hopfield neural networks, Comput. Math. Appl. 56 (2008) 1838-1844.

[8] B. Xiao, H. Meng, Existence and exponential stability of positive almost periodic solutions for high-order Hopfield neural networks, Appl. Math. Modelling 33 (2009) 532-542.

[9] J.L. Qiu, Dynamics of high-order Hopfield neural networks with time delays, Neurocomputing 73 (2010) 820-826.

[10] J.H. Park, C.H. Park, O.M. Kwon, S.M. Lee, A new stability criterion for bidirectional associative memory neural networks of neutral-type, Appl. Math. Comput. 199 (2008) 716-722.

[11] R. Rakkiyappan, P. Balasubramaniam, New global exponential stability results for neutral type neural networks with distributed time delays, Neurocomputing 71 (2008) 10391045.

[12] B. Xiao, Existence and uniqueness of almost periodic solutions for a calss of Hopfield neural networks with neutral delays, Appl. Math. Lett. 22 (2009) 528-533.

[13] R. Samidurai, S.M. Anthoni, K. Balachandran, Global exponential stability of neutraltype impulsive neural networks with discrete and distributed delays, Nonlinear Anal.: Hybrid Syst. 4 (2010) 103-112.

[14] Y.K. Li, L. Zhao, X.R. Chen, Existence of periodic solutions for neutral type cellular neural networks with delays, Appl. Math. Modelling 36 (2012) 1173-1183. 
[15] H. Zhou, Z.F. Zhou, W. Jiang, Almost periodic solutions for neutral type BAM neural networks with distributed leakage delays on time scales, Neurocomputing 157 (2015) 223-230.

[16] X. Wu, Y.N. Wang, L.H. Huang, Y. Zuo, Robust exponential stability criterion for uncertain neural networks with discontinuous activation functions and time-varying delays, Neurocomputing 73 (2010) 1265-1271.

[17] Q.T. Gan, Exponential synchronization of stochastic Cohen-Grossberg neural networks with mixed time-varying delays and reaction-diffusion via periodically intermittent control, Neural Netw. 31 (2012) 12-21.

[18] H. Zhang, J.Y. Shao, Existence and exponential stability of almost periodic solutions for CNNs with time-varying leakage delays, Neurocomputing 121 (2013) 226-233.

[19] H. Zhang, J.Y. Shao, Almost periodic solutions for cellular neural networks with timevarying delays in leakage terms, Appl. Math. Comput. 219 (24) (2013) 11471-11482.

[20] S.J. Long, Q.K. Song, X.H.Wang, D.S. Li, Stability analysis of fuzzy cellular neural networks with time delay in the leakage term and impulsive perturbations, J. Frankl. Inst. 349 (7) (2012) 2461-2479.

[21] L.J. Banu, P. Balasubramaniam, K. Ratnavelu, Robust stability analysis for discretetime uncertain neural networks with leakage time-varying delay, Neurocomputing 151 (2015) 808-816.

[22] S. Hilger, Analysis on measure chains-a unified approach to continuous and discrete calculus, Result. Math. 18 (1990) 18-56.

[23] Y.K. Li, C. Wang, X. Li, Existence and global exponential stability of almost periodic solution for high-order BAM neural networks with delays on time scales, Neural Process. Lett. 39(3) (2014) 247-268.

[24] Y.K. Li, L. Yang, Almost automorphic solution for neutral type high-order Hopfield neural networks with delays in leakage terms on time scales, Appl. Math. Comput. 242 (2014) 679-693.

[25] Y. Liu, Y.Q. Yang, T. Liang, L. Li, Existence and global exponential stability of antiperiodic solutions for competitive neural networks with delays in the leakage terms on time scales, Neurocomputing 133 (2014) 471-482.

[26] C.Y. Zhang, Pseudo almost periodic solutions of some differential equations, J. Math. Anal. Appl. 151 (1994) 62-76.

[27] E.A. Dads, K. Ezzinbi, O. Arino, Pseudo almost periodic solutions of some differential equations in a Banach space, Nonlinear Anal. TMA 28 (1997) 1141-1155. 
[28] T. Diagana, Pseudo almost periodic solutions to some differential equations, Nonlinear Anal. 60 (7)(2005) 1277-1286.

[29] T. Diagana, C.M. Mahop, G.M. N'Guérékata, Pseudo almost periodic solution to some semilinear differential equations, Math. Comput. Modelling 43 (1-2) (2006) 89-96.

[30] S. Abbas, Pseudo almost periodic sequence solutions of discrete time cellular neural networks, Nonlinear Anal. Model. Control 14 (3)(2009) 283-301.

[31] M. Pinto, Pseudo-almost periodic solutions of neutral integral and differential equations with applications, Nonlinear Anal. 72 (2010) 4377-4383.

[32] X. Chen, X. Hu, Weighted pseudo almost periodic solutions of neutral functional differential equations, Nonlinear Anal. Real World Appl. 12 (2011) 601-610.

[33] L.L. Zhang, H.X. Li, Weighted pseudo almost periodic solutions of second order neutral differential equations with piecewise constant arguments, Nonlinear Anal. 74 (2011) 67706780 .

[34] H.S. Ding, G.M. N'Guérékata, J.J. Nieto, Weighted pseudo almost periodic solutions for a class of discrete hematopoiesis model, Revista Matemática Complutense 26 (2013) 427-443.

[35] R.K. Zhuang, R. Yuan, Weighted pseudo almost periodic solutions of $n$-th order neutral differential equations with piecewise constant arguments, Acta Math. Sin. (Engl. Ser.) 30 (2014) 1259-1272.

[36] F. Chérif, Pseudo almost periodic solutions of impulsive differential equations with delay, Differ. Equ. Dyn. Syst. 22 (2014) 73-91.

[37] H.Y. Zhu, C.H. Feng, Existence and global uniform asymptotic stability of pseudo almost periodic solutions for Cohen-Grossberg neural networks with discrete and distributed delays, Math. Probl. Eng. 2014 (2014), Article ID 968404, 10 pages.

[38] L.L. Zhao, Y.K. Li, Global exponential stability of weighted pseudo-almost periodic solutions of neutral Type high-order hopfield neural networks with distributed delays, Abstr. Appl. Anal. 2014 (2014), Article ID 506256, 17 pages.

[39] B.W. Liu, Pseudo almost periodic solutions for neutral type CNNs with continuously distributed leakage delays, Neurocomputing 148 (2015) 445-454.

[40] J.X. Meng, Global exponential stability of positive pseudo-almost-periodic solutions for a model of hematopoiesis, Abstr. Appl. Anal. 2013 (2013), Article ID 463076, 7 pages.

[41] W.T. Wang, B.W. Liu, Global exponential stability of pseudo almost periodic solutions for SICNNs with time-varying leakage delays, Abst. Appl. Anal. 2014 (2014), Article ID 967328, 17 pages. 
[42] Y.K. Li, C. Wang, Pseudo almost periodic functions and pseudo almost periodic solutions to dynamic equations on time scales, Adv. Difference Equ. 2012, 2012:77.

[43] M. Bohner, A. Peterson, Dynamic Equations on Time Scales, An Introuduction with Applications, Birkhäuser, Boston, 2001.

[44] M. Bohner, A. Peterson, Advances in Dynamic Equations on Time Scales, Birkhäuser, Boston, 2003.

[45] Y.K. Li, C. Wang, Almost periodic functions on time scales and applications, Discrete Dyn. Nat. Soc. 2011 (2011), Article ID 727068, 20 pages.

[46] Y.K. Li, C. Wang, Uniformly almost periodic functions and almost periodic solutions to dynamic equations on time scales, Abstr. Appl. Anal. 2011 (2011), Article ID 341520, 22pp.

[47] I. Vasile, Fixed Point Theory: An Introduction (Dordrecht, Holland 1981). 